\title{
ON INCREASING THE EFFICIENCY OF A DISCRETE ORDINATES RADIATIVE TRANSFER METHOD WITH PERIODIC RELATIONS
}

\author{
M. P. de Abreu | ABSTRACT \\ Universidade do Estado do Rio de Janeiro \\ Instituto Politécnico \\ Departamento de Modelagem Computacional \\ Caixa Postal 97282 \\ 28601-970, Nova Friburgo, Rio de Janeiro \\ Brasil \\ deabreu@iprj.uerj.br \\ In this article, improvements in a recently developed discrete ordinates method - the two- \\ component method - are reported. The method solves conservative and non-conservative \\ radiative heat transfer problems with anisotropic scattering on a multislab domain irradiated \\ from one side with a radiation beam. The beam here consists of a monodirectional (singular) \\ stream and of a continuous (regular) distribution in angle. Specifically, the computational \\ efficiency of this two-component method has been increased with the help of new periodic \\ relations for the coupling coefficients that appear in the numerical component of the method. \\ With these periodic relations, memory usage requirement for storing the (usually large \\ number of) coupling coefficients has been halved, while saving computer time from \\ unnecessary computation of redundant coefficients. The increased efficiency of the two- \\ component method has been illustrated with numerical results and discussion of a model \\ problem in shortwave radiative transfer. \\ Keywords: radiative heat transfer, discrete ordinates, mixed beams, multislab \\ problems, computational efficiency
}

\section{NOMENCLATURE}

angular component, dimensionless

constant in the particular solution component of the intensity, $\mathrm{Wm}^{-2} \mathrm{Sr}^{-1}$

$g$

\section{Greek symbols}

expansion coefficient in the homogeneous solution component of the intensity, $\mathrm{Wm}^{-2} \mathrm{Sr}^{-1}$ Legendre component of the scattering phase function, dimensionless

$\gamma$ boundary function for the intensity, $\mathrm{Wm}^{-2} \mathrm{sr}^{-1}$

$\Delta \tau$ optical thickness, dimensionless

$\delta \quad$ Dirac distribution, dimensionless

$\theta$ coefficient in the ESGF equations, dimensionless

$\mu \quad$ cosine of the polar angle, dimensionless

$v \quad$ separation constant, dimensionless

$\tau \quad$ optical depth, dimensionless

$\phi \quad$ angular moment of the intensity, $\mathrm{Wm}^{-2}$

$\Omega \quad$ multislab domain, dimensionless

$\omega$ angular weight, dimensionless $\varpi \quad$ single scattering albedo, dimensionless

\section{Subscripts}

$i \quad$ relative to ordered set

$j \quad$ relative to layer edge and ordered set

$\ell$ relative to a component in a Legendre expansion

$m$ relative to discrete direction

$N \quad$ relative to order of quadrature set

$n$ relative to discrete direction

$p \quad$ relative to particular solution

$R \quad$ relative to right boundary and rightmost layer

$r \quad$ relative to layer number and layer edge

$t$ relative to discrete direction

$u$ relative to discrete direction

$0 \quad$ relative to left boundary

\section{Superscripts}

d relative to diffusive problem

$r \quad$ relative to layer number

$T$ relative to transpose matrix

$u$ relative to uncollided

0 relative to left boundary and zeroth order

$+\quad$ relative to downwelling heat flux

- $\quad$ relative to upwelling heat flux

\section{INTRODUCTION}

A two-component method for solving both conservative and non-conservative discrete 


\section{CIÊNCIA/SCIENCE}

ordinates $\left(\mathrm{S}_{\mathrm{N}}\right)$ radiative heat transfer problems defined on a multislab domain irradiated from one side with a beam of radiation has been recently developed by the author (de Abreu, 2003; de Abreu, 2004a). The beam is allowed to be composed of a monodirectional (singular) stream and of a continuous (regular) distribution in angle. The twocomponent method starts with a variant to the singular-regular Chandrasekhar technique (Chandrasekhar, 1950) for the decomposition of the target problem into an uncollided problem with one-sided singular boundary conditions and a diffusive problem with regular boundary conditions. Solution to the uncollided problem is fairly easily obtained but, solution to the diffusive problem is not usually so. Then, a standard $\mathrm{S}_{\mathrm{N}}$ approximation (Lewis and Miller Jr., 1993) has been considered to the diffusive problem, which has been solved with an improved spectral nodal method free from spatial truncation error (de Abreu, 2003; de Abreu, 2004a). In addition, the slabgeometry equivalence between $\mathrm{S}_{\mathrm{N}}$ and spherical harmonics $\left(\mathrm{P}_{\mathrm{N}}\right)$ formulations (Duderstadt and Martin, 1979) has been used to generate an angularly continuous approximation to the solution of the diffusive problem. Finally, uncollided and diffuse solutions have been composed to give an approximate solution to a target problem.

In this article, improvements in this twocomponent method are reported. Specifically, its computational efficiency has been increased by reducing the storage and the number of systems for the determination of the coupling coefficients in the auxiliary equations of the spectral nodal method used here for the solution of the $\mathrm{S}_{\mathrm{N}}$ version of the diffusive problem. Increase in computational efficiency is achieved by using periodic relations involving the aforementioned coupling coefficients. The increased efficiency of the method is illustrated with numerical results for a model problem in shortwave radiative transfer.

\section{TARGET PROBLEM AND ANALYSIS}

In this section, the target problem that represents the class of radiative transfer problems dealt with in this article is introduced and analyzed. Since most of the related discussion can be found in earlier work (de Abreu, 2004a; de Abreu, 2004b), presentation here will be brief. The equation of transfer with arbitrary (Legendre) order of anisotropic scattering of the form considered here is shown below:

$$
\mu \frac{\partial}{\partial \tau} I(\tau(\tau,+I(\tau(\tau,=S(\tau(\tau, \mu) \in \Omega \equiv[\tau, \tau]=,-1 \leq \mu \leq 1,
$$

where $\tau$ is the optical variable defined on a multislab domain $\Omega$ with no reemitting boundaries denoted by $\tau_{0}$ (left) and $\tau_{\mathrm{R}}$ (right), respectively; $\mu$ is the cosine of the polar angle defined by the direction of the propagating radiation and the positive $\tau$-axis. The quantity $\mathrm{I}(\tau, \mu)$ is the frequency-integrated intensity of the radiation field in the $\mu$ direction at optical depth $\tau$ and $S(\tau, \mu)$ is the scattering source function given by:

$$
S\left(\tau ( \tau , = \frac { \varpi ( \tau \tau } { 2 } \sum _ { \ell = 0 } ^ { \infty } ( 2 \ell + 1 ) \beta _ { \ell } ( \tau \tau ) ) _ { \ell } \left(\mu \mu \int_{-1}^{l} d \mu^{\prime} P_{\ell}\left(\mu^{\prime}\right) I\left(\tau I \mu^{\prime}\right)\right.\right.
$$

The quantity $\varpi(\tau)$ is the single scattering albedo at depth $\tau ;(2 \ell+1) \beta_{\ell}(\tau)$ is the $\ell$ th-order component of the Legendre expansion of the scattering phase function and $\mathrm{P}_{\ell}(\mu)$ denotes the $\ell$ th-degree Legendre polynomial. We assume that the multislab domain $\Omega$ consists of $\mathrm{R}$ contiguous and disjoint layers of homogeneous material each, i.e. the quantities $\varpi(\tau)$ and $\beta_{\ell}(\tau)$, for all $\ell$, are piecewise constant functions of $\tau$ on $\Omega$. Equation (1) is subject to the boundary conditions

$$
\begin{gathered}
I\left(\tau_{0}, \mu \mu=I_{0} \delta\left(\mu-\mu_{0}\right)+\gamma_{0}(\mu \mu) \mu>0, \mu_{0}>0,\right. \\
I\left(\tau_{R},-\mu\right)=0, \mu>0
\end{gathered}
$$

where $\mathrm{I}_{0}$ is a non-negative real; $\mu_{0}$ is the cosine of the polar angle defining the direction of incidence of the monodirectional component of the beam of radiation upon the left boundary of the multislab domain $\Omega$; the symbol $\delta$ is to denote a Dirac distribution and $\gamma_{0}(\mu), \mu>0$, is a nonnegative function of $\mu$ representing the angularly continuous part of the incident beam of radiation.

Equations (1)-(3) define the (mathematical) target problem representing the class of radiative transfer problems dealt with in this article.

Following a decomposition technique introduced by Chandrasekhar (1950) in solving a basic problem in radiative transfer in planetary atmospheres, the target problem (Eqs. 1-3) has been decomposed into the uncollided problem 


\section{CIÊNCIA/SCIENCE}

$$
\mu \frac{\partial}{\partial \tau} I^{u}\left(\tau \tau, \mu+I^{u}(\tau \tau, \mu=0, \tau \in \Omega,-1 \leq \mu \leq 1,\right.
$$

with the left singular boundary conditions

$$
I^{u}\left(\tau_{0}, \mu \mu=I_{0} \delta\left(\mu-\mu_{0}\right) ; I^{u}\left(\tau_{R^{\prime}}-\mu\right)=0, \mu>0, \mu_{0}>0,\right.
$$

and the diffusive problem

$$
\begin{aligned}
& \mu \frac{\partial}{\partial \tau} I^{d}\left(\tau \tau, \mu+I^{d}(\tau \tau, \mu=\right. \\
& \left.\frac{\varpi(\tau \tau}{2} \sum_{\ell=0}^{\infty}(2 \ell+1) \beta_{\ell}(\tau \tau)\right)_{\ell}\left(\mu \mu \int_{-1}^{l} d \mu^{\prime} P_{\ell}\left(\mu^{\prime}\right) I^{d}\left(\tau \tau \mu^{\prime}\right)\right. \\
& +s^{u}(\tau \tau, \mu) \tau \in \Omega,-1 \leq \mu \leq 1,
\end{aligned}
$$

with the regular boundary conditions

$$
I^{d}\left(\tau_{0}, \mu \mu=\gamma_{0}(\mu \mu) I^{d}\left(\tau_{R},-\mu\right)=0, \mu>0,\right.
$$

So that $I\left(\tau\left(\tau,=I^{u}\left(\tau \tau, \mu+I^{d}(\tau \tau, \mu) \tau_{0} \leq \tau \leq \tau_{R^{\prime}},-1 \leq \mu \leq 1\right.\right.\right.$. The quantity

$$
s^{u}\left(\tau \tau, \mu \equiv \frac{\varpi(\tau \tau}{2} \sum_{\ell=0}^{\infty}(2 \ell+1) \beta_{\ell}(\tau \tau)\right)_{\ell}\left(\mu \mu \int_{-l}^{l} d \mu^{\prime} P_{\ell}\left(\mu^{\prime}\right) I^{u}\left(\tau \pi \ell^{\prime}\right)\right.
$$

in Eq. (6) is a depth-dependent anisotropic source given in terms of the solution $I^{\mathrm{u}}(\tau, \mu)$ to the uncollided transport problem (Eqs. 4-5).

Solution to this problem is fairly easily obtained (de Abreu, 2004a) and has the closed form

$$
\left\{\begin{array}{l}
I^{u}\left(\tau \tau, \mu=I_{0} \delta\left(\mu-\mu_{0}\right) \exp \left[-\frac{1}{\mu}\left(\tau-\tau_{0}\right)\right],\right. \\
I^{u}(\tau \tau-\mu)=0, \tau \in \Omega, \mu>0, \mu_{0}>0 .
\end{array}\right.
$$

Substituting the closed form solution (Eq. 9) into the source (Eq. 8) yields:

$$
\begin{aligned}
& s^{u}\left(\tau \tau, \mu=I_{0} \exp \left[-\frac{1}{\mu_{0}}\left(\tau-\tau_{0}\right)\right] \frac{\varpi(\tau \tau}{2} *\right. \\
& \sum_{\ell=0}^{\infty}(2 \ell+1) \beta_{\ell}(\tau \tau){ }_{\ell}(\mu \mu)_{\ell}\left(\mu_{0}\right) .
\end{aligned}
$$

Decomposing the multislab domain $\Omega$ into its $R$ contiguous and disjoint homogeneous subdomains (layers) yieds the local (layer-level) diffusive equations:

$$
\begin{aligned}
& \mu \frac{\partial}{\partial \tau} I_{r}^{d}\left(\tau \tau, \mu+I_{r}^{d}(\tau \tau, \mu=\right. \\
& \frac{\varpi_{r}}{2} \sum_{\ell=0}^{\infty}(2 \ell+1) \beta_{\ell, r} P_{\ell}\left(\mu \int_{-l}^{l} d \mu^{\prime} P_{\ell}\left(\mu^{\prime}\right) I_{r}^{d}\left(\tau \pi \ell^{\prime}\right)+s_{r}^{u}(\tau \tau, \mu)\right. \\
& \tau_{r-1} \leq \tau \leq \tau_{r}, r=1: R,-1 \leq \mu \leq 1,
\end{aligned}
$$

with $I_{1}^{d}\left(\tau_{0}, \mu \mu=\gamma_{0}(\mu \mu) I_{R}^{d}\left(\tau_{R},-\mu\right)=0, \mu>0\right.$, and with intensity continuity conditions at layer interfaces, i.e.,

$$
I_{j}^{d}\left(\tau_{j}, \mu \mu=I_{j+1}^{d}\left(\tau_{j}, \mu \mu\right)-1 \leq \mu \leq 1, j=1: R-1,\right.
$$

where $\tau_{\mathrm{j}}, \mathrm{j}=1: R-1$, is to denote the $\mathrm{jth}$ layer interface. Considering a standard $S_{\mathrm{N}}$ approximation (Lewis and Miller, 1993) to the local equations (Eq. 11) in the form:

$$
\begin{aligned}
& \mu_{m} \frac{d}{d \tau} I_{r, m}^{d}\left(\tau \tau+I_{r, m}^{d}(\tau \tau=\right. \\
& \frac{\varpi_{r}}{2} \sum_{\ell=0}^{L_{r}}(2 \ell+1) \beta_{\ell, r} P_{\ell}\left(\mu_{m}\right) \sum_{n=l}^{N} \omega_{n} P_{\ell}\left(\mu_{n}\right) I_{r, n}^{d}(\tau \tau \\
& +s_{r, m}^{u}(\tau \tau) m=1: N, \tau_{r-1} \leq \tau \leq \tau_{r}, r=1: R,
\end{aligned}
$$

where $I_{r, m}^{d}\left(\tau \tau \cong I_{r}^{d}\left(\tau \tau,{ }_{m}\right)\right.$ and $s_{r, m}^{u}\left(\tau \tau \cong s_{r}^{u}\left(\tau \tau,{ }_{m}\right)\right.$.

Solution to the $S_{\mathrm{N}}$ equations (Eq. 13) can be expressed in terms of a homogeneous solution and of a particular solution in the vector form

$$
I_{r}^{d}\left(\tau \tau=\sum_{i=1}^{N} \alpha_{r, i} I_{r, i}^{d}\left(\tau \tau+I_{r, p}^{d}(\tau \tau) \tau_{r-1} \leq \tau \leq \tau_{r}\right.\right.
$$

where

$$
I_{r}^{d}\left(\tau \tau \equiv \left[I_{r, 1}^{d}(\tau \tau),{ }_{r, 2}^{d}(\tau \tau) \ldots, I_{r, N}^{d}(\tau \tau)^{T}\right.\right.
$$

$\alpha_{\mathrm{r}, \mathrm{i}}, \mathrm{i}=1: N$, are (open) scalars depending upon boundary/interface conditions;

$$
\begin{aligned}
& I_{r, i}^{d}\left(\tau \tau \equiv \left[I_{r, i, I}^{d}(\tau \tau){ }_{r, i, 2}^{d}(\tau \tau) \ldots, I_{r, i, N}^{d}(\tau \tau)^{T},\right.\right. \\
& i=1: N, \tau_{r-1} \leq \tau \leq \tau_{r},
\end{aligned}
$$




\section{CIÊNCIA/SCIENCE}

are the elements of a vector basis for the null space of the local $\mathrm{S}_{\mathrm{N}}$ radiative transfer operator

$$
\left[\mu_{m} \frac{d}{d \tau}+1\right](\bullet)-\frac{\varpi_{r}}{2} \sum_{\ell=0}^{L_{r}}(2 \ell+1) \beta_{\ell, r} P_{\ell}\left(\mu_{m}\right) \sum_{n=l}^{N} \omega_{n} P_{\ell}\left(\mu_{n}\right)(\bullet),
$$

and

$$
I_{r, p}^{d}\left(\tau \tau \equiv \left[I_{r, p, I}^{d}(\tau \tau){ }_{r, p, 2}^{d}(\tau \tau) \ldots, I_{r, p, N}^{d}(\tau \tau)^{T} .\right.\right.
$$

The entries of vector (Eq. 16) are either exponentials given by:

$$
\begin{aligned}
& I_{r, i, m}^{d}\left(\tau \tau=a_{r, m}\left(v_{r, i}\right) \exp \left(\frac{\tau-\tau_{r, i}}{v_{r, i}}\right),\right. \\
& \tau_{r-1} \leq \tau \leq \tau_{r}, i=1: N, m=1: N,
\end{aligned}
$$

or first-degree polynomials in $\tau$ of the form:

$$
\frac{\left(\tau_{r}-\tau\right)}{\Delta \tau_{r}}+\frac{\mu_{m}}{\Delta \tau_{r}\left(1-\beta_{1, r}\right)}, m=1: N
$$

and

$$
\frac{\left(\tau-\tau_{r-1}\right)}{\Delta \tau_{r}}-\frac{\mu_{m}}{\Delta \tau_{r}\left(1-\beta_{1, r}\right)}, m=1: N,
$$

with $\Delta \tau_{\mathrm{r}} \equiv \tau_{\mathrm{r}}-\tau_{\mathrm{r}-1}$ and $\left|\beta_{1, \mathrm{r}}\right|<1$.

It should be noted that the quantities $\tau_{\mathrm{r}, \mathrm{i}}, \mathrm{i}=$ $1: \mathrm{N}$, in the exponentials (Eq. 19) are appropriate optical depths and $v_{\mathrm{r}, \mathrm{i}}$ and $\mathrm{a}_{\mathrm{r}, \mathrm{m}}\left(v_{\mathrm{r}, \mathrm{i}}\right)$, are the separation constants and the angular components of the exponential solutions (Eq. 19), respectively.

Polynomials (Eqs. 20-21) were used as elementary solutions of the homogeneous version of Eq. (13) for the degenerate case of conservative layers (Chandrasekhar, 1950; de Abreu, 2004a). A numerical scheme for determining the separation constants and angular components is fully described in an earlier work of the author (de Abreu, 1998), while the optical depths $\tau_{\mathrm{r}, \mathrm{i}}, \mathrm{i}=1: N$, may be found in a more recent work (de Abreu, 2004a).

The entries of vector (18) are given by the exponential functions

$$
I_{r, p, m}^{d}\left(\tau \tau=f_{r, m} \exp \left(-\frac{\tau}{\mu_{0}}\right), \tau_{r-1} \leq \tau \leq \tau_{r}, m=1: N .\right.
$$

The determination of the constants $\mathrm{f}_{\mathrm{r}, \mathrm{m}}, \mathrm{m}=1: \mathrm{N}$, in the exponential functions (Eq. 22) is reported in detail by Siewert (2000).

\section{A TWO-COMPONENT METHOD}

The method described in this section is a conjugation of basic relations from more general results in the theory of radiation transport and spectral nodal methods recently developed by the present author and former collaborators.

The approximate solution to the target problem proposed here is a distribution on $\tau$ and $\mu$ of the form

$$
I_{N}\left(\tau \tau, \mu=I^{u}\left(\tau \tau, \mu+I_{N-I}^{d}(\tau \tau, \mu) \tau_{0} \leq \tau \leq \tau_{R},-1 \leq \mu \leq 1,\right.\right.
$$

where the second term on the right-hand side denotes the spherical harmonics $\left(\mathrm{P}_{\mathrm{N}-1}\right)$ approximation (Duderstadt and Martin, 1979; Lewis and Miller Jr., 1993) to the solution of the local diffusive equations (Eq. 11), which is given by:

$$
\begin{aligned}
& I_{N-I}^{d}\left(\tau \tau, \mu=\sum_{\ell=0}^{N-l} \frac{(2 \ell+1)}{2} \varphi_{r, \ell}^{d}\left(\tau \tau P_{\ell}(\mu \mu\right.\right. \\
& \tau_{r-1} \leq \tau \leq \tau_{r},-1 \leq \mu \leq 1, r=1: R,
\end{aligned}
$$

The quantities

$$
\varphi_{r, \ell}^{d}\left(\tau \tau=\sum_{i=l}^{N} \omega_{t} P_{\ell}\left(\mu_{t}\right) I_{r, t}^{d}\left(\tau \tau, \tau_{r-1} \leq \tau \leq \tau_{r}, r=1: R,\right.\right.
$$

are the $P_{\mathrm{N}-1}$ angular moments of the diffuse component of the intensity.

As the name implies, the two-component method has two ingredients: a numerical component and an analytical component. The numerical component is to provide layer-average

$$
\bar{I}_{r, m}^{d} \equiv \frac{1}{\Delta \tau_{r}} \int_{\tau_{r-1}}^{\tau_{r}} I_{r, m}^{d}(\tau \tau d \tau, m=1: N, r=1: R,
$$

and layer-edge values for the entries of the $\mathrm{S}_{\mathrm{N}}$ solution vector (Eq. 15) without having to determine the scalars $\alpha_{\mathrm{r}, \mathrm{i}}, \mathrm{r}=1: \mathrm{R}, \mathrm{i}=1: \mathrm{N}$. The numerical component is thus suited to radiative transfer problems where the quantities of interest are, for example, the angular distribution of 


\section{CIÊNCIA/SCIENCE}

radiation leaving the multislab domain and angleintegrated layer-edge quantities such as radiative heat fluxes (Chandrasekhar, 1950; Thomas and Stamnes, 1999).

The analytical component of the twocomponent method is to reconstruct the approximate solution (Eq. 24) by solving a system of linear algebraic equations for the scalars $\alpha_{\mathrm{r}, \mathrm{i}}$ in the $\mathrm{S}_{\mathrm{N}}$ solution (Eq. 15). Inputs to the system are layer-edge values supplied by the numerical component. The analytical component is to be applied when the intensity of the radiation field $\mathrm{I}_{\mathrm{N}}(\tau, \mu)$ at any depth $\tau$ and direction $\mu$ is sought. Both components are briefly described below.

The numerical component of the twocomponent method is a numerical method designed for solving the $\mathrm{S}_{\mathrm{N}}$ diffusive problem (Eq. 13) with no optical truncation error. It is an extension to anisotropic scattering of arbitrary order and depth-dependent anisotropic sources of the spectral Green's function (SGF) method for neutron transport problems (Barros and Larsen, 1990). For this reason, it is referred to as the extended spectral Green's function (ESGF) method.

The ESGF method has two main ingredients: one is standard and the other is nonstandard. The standard ingredient is the derivation of radiative balance equations on each layer of the multislab domain $\Omega$, i.e.,

$$
\begin{aligned}
& \frac{\mu_{m}}{\Delta \tau_{r}}\left(I_{r, m}^{d}-I_{r-1, m}^{d}\right)+\bar{I}_{r, m}^{d}= \\
& \frac{\varpi_{r}}{2} \sum_{\ell=0}^{L_{r}}(2 \ell+1) \beta_{\ell, r} P_{\ell}\left(\mu_{m}\right) \sum_{n=1}^{N} \omega_{n} P_{\ell}\left(\mu_{n}\right) \bar{I}_{r, n}^{d}+ \\
& \bar{s}_{r, m}^{u}, r=1: R, m=1: N,
\end{aligned}
$$

where

$$
\begin{aligned}
& \bar{s}_{r, m}^{u} \equiv \frac{1}{\Delta \tau_{r}} \int_{\tau_{r-1}}^{\tau_{r}} d \tau s_{r, m}^{u}(\tau \tau= \\
& s_{r, m}^{0} \frac{\mu_{0}}{\Delta \tau_{r}}\left[\exp \left(-\frac{\tau_{r-1}}{\mu_{0}}\right)-\exp \left(-\frac{\tau_{r}}{\mu_{0}}\right)\right],
\end{aligned}
$$

is the discretized source term. The non-standard ingredient is the derivation of the ESGF auxiliary equations

$$
\begin{aligned}
& \bar{I}_{r, m}^{d}=\sum_{u=1}^{N / 2} \theta_{r, m, u} I_{r-1, u}^{d}+\sum_{u=N / 2+1}^{N} \theta_{r, m, u} I_{r, u}^{d}+g_{r, m}, \\
& r=1: R, m=1: N,
\end{aligned}
$$

where the layer-dependent coefficients $\theta_{\mathrm{r}, \mathrm{m}, \mathrm{u}}$ and $\mathrm{g}_{\mathrm{r}, \mathrm{m}}$ are determined so that the analytical solution (Eq. 14) does satisfy the ESGF auxiliary equations (Eq. 29), for arbitrary scalars $\alpha_{\mathrm{r}, \mathrm{i}}$ and for the entries of vector (Eq. 18) given by the exponential functions (Eq. 22). Discussion of the ESGF auxiliary equations (Eq. 29) is left to the next section.

Equations (27) and (29) constitute the system of discretized equations of the ESGF method. Solution methods for this system are discussed elsewhere (Barros and Larsen, 1990).

The analytical component of our twocomponent method is a local (layer-level) analytical reconstruction scheme of the approximate solution (Eq. 24). It is based upon solving a local system of $N$ linear algebraic equations whose unknowns are the scalars $\alpha$ r, i, r fixed, $\mathrm{i}=1: N$. Inputs to the system are the layer-edge intensities that are incident upon the layer of interest (de Abreu and Barros, 1994). These layer-edge intensities are supplied by the ESGF method. More details can be found in a recent work of the author (de Abreu, 2004a).

\section{INCREASING THE EFFICIENCY WITH PERIODIC RELATIONS}

The coefficients $\theta_{\mathrm{r}, \mathrm{m}, \mathrm{u}}$ and $\mathrm{g}_{\mathrm{r}, \mathrm{m}}, \mathrm{r}=1: R, \mathrm{~m}=$ $1: N, \mathrm{u}=1: N$, in the ESGF equations (Eq. 29) follow from a standing condition - the open form (Eq. 14) satisfies Eqs. (29) for arbitrary scalars $\alpha_{\mathrm{r}, \mathrm{i}}, \mathrm{r}=$ $1: R, \mathrm{i}=1: N$, and arbitrary constants $\mathrm{f}_{\mathrm{r}, \mathrm{m}}, \mathrm{r}=1: R, \mathrm{~m}$ $=1: N$, in the exponential functions (Eq. 22). From this condition (de Abreu, 2003; de Abreu, 2004a), the coefficients $\mathrm{g}_{\mathrm{r}, \mathrm{m}}, \mathrm{r}=1: R, \mathrm{~m}=1: N$, can be found to be given by:

$$
\begin{aligned}
& g_{r, m}=\frac{\mu_{0} f_{r, m}}{\Delta \tau_{r}}\left[\exp \left(-\frac{\tau_{r-1}}{\mu_{0}}\right)-\exp \left(-\frac{\tau_{r}}{\mu_{0}}\right)\right]- \\
& {\left[\exp \left(-\frac{\tau_{r-1}}{\mu_{0}} \sum_{u=1}^{N / 2} \theta_{r, m, u} f_{r, u}+\exp \left(-\frac{\tau_{r}}{\mu_{0}}\right) \sum_{u=N / 2+1}^{N} \theta_{r, m, l} f_{r, u}\right],\right.}
\end{aligned}
$$

$m=1: N$,

and the $\mathrm{N}$ coefficients $\theta_{\mathrm{r}, \mathrm{m}, \mathrm{u}}$ ( $\mathrm{r}$ and $\mathrm{m}$ fixed, $\mathrm{u}$ varying 


\section{CIÊNCIA/SCIENCE}

from 1 to $N$ ) are found to satisfy the system of $\mathrm{N}$ linear algebraic equations

$$
\begin{aligned}
& \frac{v_{r, j} a_{r, m}\left(v_{r, j}\right)}{\Delta \tau_{r}} * \\
& {\left[\exp \left(\frac{\tau_{r}-\tau_{r, j}}{v_{r, j}}\right)-\exp \left(\frac{\tau_{r-1}-\tau_{r, j}}{v_{r, j}}\right)\right]=} \\
& \exp \left(\frac{\tau_{r-1}-\tau_{r, j}}{v_{r, j}}\right) \sum_{u=1}^{N / 2} \theta_{r, m, u} a_{r, u}\left(v_{r, j}\right)+ \\
& \exp \left(\frac{\tau_{r}-\tau_{r, j}}{v_{r, j}}\right) \sum_{u=N / 2+1}^{N} \theta_{r, m, u} a_{r, u}\left(v_{r, j}\right), j=1: N,
\end{aligned}
$$

for a non-conservative layer $\left(0 \leq \varpi_{\mathrm{r}}<1\right)$, and the system

$$
\begin{aligned}
& {\left[\frac{1}{2}+\frac{\mu_{m}}{\Delta \tau_{r}\left(1-\beta_{1, r}\right)}\right]=} \\
& \sum_{u=1}^{N / 2} \theta_{r, m, u}\left[1+\frac{\mu_{u}}{\Delta \tau_{r}\left(1-\beta_{1, r}\right)}\right]+ \\
& \sum_{u=N / 2+1}^{N} \theta_{r, m, u}\left[\frac{\mu_{u}}{\Delta \tau_{r}\left(1-\beta_{l, r}\right)}\right], \\
& {\left[\frac{1}{2}-\frac{\mu_{m}}{\Delta \tau_{r}\left(1-\beta_{1, r}\right)}\right]=} \\
& \sum_{u=1}^{N / 2} \theta_{r, m, u}\left[-\frac{\mu_{u}}{\Delta \tau_{r}\left(1-\beta_{l, r}\right)}\right]+ \\
& \sum_{u=N / 2+1}^{N} \theta_{r, m, u}\left[1-\frac{\mu_{u}}{\Delta \tau_{r}\left(1-\beta_{l, r}\right)}\right]
\end{aligned}
$$

and

$$
\begin{aligned}
& a_{r, m}\left(v_{r, j}\right) \frac{v_{r, j}}{\Delta \tau_{r}} * \\
& {\left[\exp \left(\frac{\tau_{r}-\tau_{r, j}}{v_{r, j}}\right)-\exp \left(\frac{\tau_{r-1}-\tau_{r, j}}{v_{r, j}}\right)\right]=} \\
& \exp \left(\frac{\tau_{r-1}-\tau_{r, j}}{v_{r, j}}\right) \sum_{u=1}^{N / 2} \theta_{r, m, u} a_{r, u}\left(v_{r, j}\right)+ \\
& \exp \left(\frac{\tau_{r}-\tau_{r, j}}{v_{r, j}}\right) \sum_{u=N, 2+1}^{N} \theta_{r, m, u} a_{r, u}\left(v_{r, j}\right), j=3: N,
\end{aligned}
$$

for a conservative one $\left(\varpi_{\mathrm{r}}=1\right)$. Upon substitution of the exponential solutions (Eq. 19) into the homogeneous version of Eqs. (13) and from a parity analysis of the resulting equations (Siewert, 2000; de Abreu, 2004a; de Abreu, 2004b), it is not difficult to show that the constants $v_{\mathrm{r}, \mathrm{i}}$ appear in \pm pairs of numbers and that the angular components satisfy the relation $\mathrm{a}_{\mathrm{r}, \mathrm{m}}\left(v_{\mathrm{r}, \mathrm{i}}\right)=\mathrm{a}_{\mathrm{r}-\mathrm{m}}\left(v_{\mathrm{r}, \mathrm{i}}\right)$, for all $\mathrm{r}, \mathrm{m}$ and $\mathrm{i}$, where the lowercase subscripts $-\mathrm{m}$ and $-\mathrm{i}$ are to denote the discrete direction $-\mu_{\mathrm{m}}$ and the separation constant $-v_{\mathrm{r}, \mathrm{i}}$, respectively. Next, a parity analysis of the systems of Eqs. (31) and (32) with the help of the above results is performed, beginning with Eq. (31) for non-conservative layers. Let $m$ vary only from 1 to $\mathrm{N} / 2$ in Eq. (31), so that we may licitly define a system for fixed $\mathrm{r}$ and $\mathrm{m}+\mathrm{N} / 2(\leq$ $\mathrm{N})$. Using the above relation for the angular components, and considering the parity of the separation constants, the system for fixed $r$ and $(\mathrm{m}+\mathrm{N} / 2)$ can be written in the form:

$$
\begin{aligned}
& \frac{v_{r,-j} a_{r, m+N / 2}\left(v_{r,-j}\right)}{\Delta \tau_{r}} * \\
& {\left[\exp \left(\frac{\tau_{r}-\tau_{r,-j}}{v_{r,-j}}\right)-\exp \left(\frac{\tau_{r-1}-\tau_{r,-j}}{v_{r,-j}}\right)\right]=} \\
& \exp \left(\frac{\tau_{r-1}-\tau_{r,-j}}{v_{r,-j}}\right) \sum_{u=1}^{N / 2} \theta_{r, m+N / 2, u} a_{r, u}\left(v_{r,-j}\right)+ \\
& \exp \left(\frac{\tau_{r}-\tau_{r,-j}}{v_{r,-j}}\right) \sum_{u=N / 2+1}^{N} \theta_{r, m+N / 2, u} a_{r, u}\left(v_{r,-j}\right), j=1: N .
\end{aligned}
$$

The optical depths $\tau_{\mathrm{r}, \mathrm{j}}, \mathrm{j}=1: N$, are chosen (de Abreu, 2004a; de Abreu, 2004b) so that

$$
\frac{\tau_{r-1}-\tau_{r,-j}}{v_{r,-j}}=\frac{\tau_{r}-\tau_{r, j}}{v_{r, j}},
$$

implying that

$$
\frac{\tau_{r}-\tau_{r,-j}}{v_{r,-j}}=\frac{\tau_{r-1}-\tau_{r, j}}{v_{r, j}} .
$$

Upon substitution of Eqs. (34) and (35) into the system of Eq. (33), and noting that $\mathrm{a}_{\mathrm{r}, \mathrm{m}+\mathrm{N} / 2}\left(v_{\mathrm{r}, \mathrm{j}}\right)$ $=\mathrm{a}_{\mathrm{r}, \mathrm{m}}\left(v_{\mathrm{r}, \mathrm{j}}\right)$ and that $\mathrm{a}_{\mathrm{r}, \mathrm{u}}\left(v_{\mathrm{r}, \mathrm{j}}\right)=\mathrm{a}_{\mathrm{ru}}\left(v_{\mathrm{r}, \mathrm{j}}\right)$, the system of Eq. (33) can be written in the form: 


$$
\begin{aligned}
& \frac{v_{r, j} a_{r, m}\left(v_{r, j}\right)}{\Delta \tau_{r}} * \\
& {\left[\exp \left(\frac{\tau_{r}-\tau_{r, j}}{v_{r, j}}\right)-\exp \left(\frac{\tau_{r-1}-\tau_{r, j}}{v_{r, j}}\right)\right]=} \\
& \exp \left(\frac{\tau_{r-1}-\tau_{r, j}}{v_{r, j}}\right) \sum_{u=1}^{N / 2} \theta_{r, m+N /, u+N / 2} a_{r, u}\left(v_{r, j}\right)+ \\
& \exp \left(\frac{\tau_{r}-\tau_{r, j}}{v_{r, j}}\right) \sum_{u=N / 2+1}^{N} \theta_{r, m+N /, u-N / 2} a_{r, u}\left(v_{r, j}\right), \\
& j=1: N .
\end{aligned}
$$

Termwise comparison of systems of Eqs. (31) and (36) leads to the periodic relations of period $\mathrm{N} / 2$

$$
\begin{aligned}
& \theta_{\rho, \mu, v}=\theta_{\rho, \mu+N / 2, v+N / 2} \\
& \mu=1: N: N h=1: N: N,
\end{aligned}
$$

and

$$
\begin{aligned}
& \theta_{r, m, u}=\theta_{r, m+N / 2, u-N / 2}, \\
& m=1: N / 2, u=N / 2+1: N,
\end{aligned}
$$

for the coupling coefficients in Eqs. (29). The periodic relations (Eqs. 37 and 38) show that the coupling coefficients $\theta_{\mathrm{r}, \mathrm{m}, \mathrm{u}}$, for $\mathrm{m}=N / 2+1: N$, correspond to those for $m=1: N / 2$. Since the coefficients $\theta_{\mathrm{r}, \mathrm{m}, \mathrm{u}}$ are solutions to systems of linear algebraic equations for fixed $\mathrm{r}$ and $\mathrm{m}$, the $N / 2$ systems associated with $\mathrm{m}=N / 2+1: N$ do not need to be solved, for their solutions are corresponding solutions to systems associated with $\mathrm{m}$ from 1 to N/2. Therefore, the periodic relations (Eqs. 37 and 38) have two attractive features. First, they reduce computer memory requirements, for half of the coefficients $\theta_{\mathrm{r}, \mathrm{m}, \mathrm{u}}(\mathrm{m}=N / 2+1: N)$ does not need to be stored. Second, they save computer execution time, since there is no need to solve systems for determining the coefficients $\theta_{\mathrm{r}, \mathrm{m}, \mathrm{u}}$ for $\mathrm{m}=N / 2+1: N$. So, the periodic relations (Eqs. 37 and 38) are likely to increase the computational efficiency of our twocomponent method. The same periodic relations can be obtained from termwise inspection of the system of Eq. (32) and the conservative counterpart of the system of Eq. (36).

\section{A TEST PROBLEM}

The increased efficiency of the twocomponent method is illustrated with numerical results for a test problem relevant to the transfer of shortwave radiation in a vertically heterogeneous atmosphere. It should be noticed that the numerical results reported here come from the execution of a FORTRAN program on an IBM-compatible PC (1.4 GHz-clock Intel Pentium 4 processor and 256 Mbytes of RAM) running on GNU/Linux, version 0.2. The executable file has been generated with the g77 GNU Fortran package, release 2.95. The execution (CPU) times reported here were generated with the TIME GNU internal routine, option $-\mathrm{S}$.

The test problem is based on a six-layer model for a stratified atmosphere described in a work of Devaux et al. (1979). Each of the six layers has the same scattering law but the single scattering albedo is allowed to be different in each layer. The optical thickness $\Delta \tau_{\mathrm{r}}$ and single scattering albedo $\varpi_{\mathrm{r}}$ for each layer are provided in Tab. 1. The scattering law is approximated by the $L=8$ scattering phase function data given in Tab. 2. The atmosphere is illuminated with a mixed beam having a normally incident component and a linearly anisotropic diffuse component. The boundary data for this six-layer model problem are $\tau_{0}=0, \tau_{6}=21, \quad I_{0}=0.5, \mu_{0}=1$ and $\gamma_{0}()=\mu, \mu>0$.

In Tab. 3, the layer-edge results for converged $S_{200}$ downward $\left(\mathrm{q}^{+}\right)$and upward $(\mathrm{q}-)$ radiative heat fluxes (Chandrasekhar, 1950; Thomas and Stamnes, 1999) are presented. Since no approximation has been introduced in the derivation of the periodic relations reported in the previous section, the numerical results in Tab. 3 are, as expected, the same as those tabulated in de Abreu (2003).

Table 1. Layer thickness and single scattering albedo

\begin{tabular}{|c|c|c|}
\hline $\mathrm{r}$ & $\Delta \tau_{\mathrm{r}}$ & $\varpi_{\mathrm{r}}$ \\
\hline 1 & 1.0 & 1.0 \\
\hline 2 & 2.0 & 0.70 \\
\hline 3 & 3.0 & 0.75 \\
\hline 4 & 4.0 & 0.80 \\
\hline 5 & 5.0 & 0.85 \\
\hline 6 & 6.0 & 0.90 \\
\hline
\end{tabular}


CIÊNCIA/SCIENCE

Table 2. Scattering phase function data

\begin{tabular}{|c|c|}
\hline$\ell$ & $(2 \ell+1) \beta_{\ell}$ \\
\hline 0 & 1 \\
\hline 1 & 2.00916 \\
\hline 2 & 1.56339 \\
\hline 3 & 0.67407 \\
\hline 4 & 0.22215 \\
\hline 5 & 0.04725 \\
\hline 6 & 0.00671 \\
\hline 7 & 0.00068 \\
\hline 8 & 0.00005 \\
\hline
\end{tabular}

Table 3. Results for radiative heat fluxes $\left(\mathrm{Wm}^{-2}\right)$

\begin{tabular}{|c|c|c|}
\cline { 2 - 3 } \multicolumn{1}{c|}{} & $\mathrm{q}_{200}^{+}\left(\tau_{\mathrm{j}}\right)$ & $\mathrm{q}_{200}^{-}\left(\tau_{\mathrm{j}}\right)$ \\
\hline$\tau_{0}=0$ & 5.235972 & 0.994869 \\
\hline$\tau_{1}=1$ & 4.671763 & 0.430660 \\
\hline$\tau_{2}=3$ & 1.481298 & 0.168251 \\
\hline$\tau_{3}=6$ & 0.309292 & 0.046167 \\
\hline$\tau_{4}=10$ & $4.7609 \times 10^{-2}$ & $9.4260 \times 10^{-3}$ \\
\hline$\tau_{5}=15$ & $6.4262 \times 10^{-3}$ & $1.7057 \times 10^{-3}$ \\
\hline$\tau_{6}=21$ & $8.3764 \times 10^{-4}$ & 0 \\
\hline
\end{tabular}

The expected behavior of both downward and upward shortwave radiative fluxes - a decreasing function of optical depth - for a stratified planetary atmosphere with a dark surface can be seen in Fig. 1. This happened because the atmosphere is a purely absorbing/scattering participating medium for shortwave radiation.

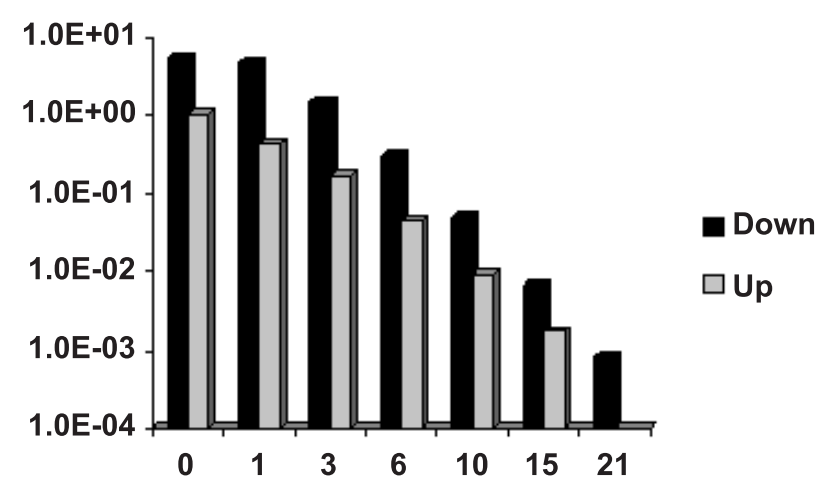

Figure 1. Downward and upward radiative fluxes $\left(\mathrm{Wm}^{-2}\right)$ through the stratified atmosphere

In Table 4, computer memory and execution times for the runs with (case 1) and without (case 2) the periodic relations (Eqs. 37 and 38) are shown. It is apparent from the savings in computer memory and execution time that the periodic relations increased the computational efficiency of the computer code version of the twocomponent method. The reduction in execution time is relatively modest, as compared to the memory one, because a considerable fraction of the CPU time is used up for computing the separation constants and the angular components in the exponentials (Eq. 19).

Table 4. Computer memory and execution time

\begin{tabular}{|c|c|c|}
\cline { 2 - 3 } \multicolumn{1}{c|}{} & Memory (Kbytes) & CPU (second) \\
\hline Case 1 & 184.7 & 115.5 \\
\hline Case 2 & 316.7 & 146.4 \\
\hline
\end{tabular}

\section{CONCLUDING REMARKS}

The periodic relations, Eqs. (37) and (38), increased the computational efficiency of the twocomponent method without degrading its numerical accuracy. These periodic relations are exact in the following sense: if the $S_{N}$ equations (Eq. 13) were to exactly describe the transport processes for the diffuse component of the radiation in the multislab medium, then the two-component method would generate exact solutions for the diffuse component of the intensity of the radiation field, with or without the periodic relations.

The periodic relations neither improve nor corrupt the numerical results generated by the twocomponent method. It has also been noted that the periodic relations are in close connection to the concept of discrete Green's functions and response matrices for boundary layer sources (Barros and Larsen, 1990; de Abreu, 2004b). This will be addressed in a forthcoming article.

\section{REFERENCES}

Barros, R. C., and Larsen, E. W., 1990, A Numerical Method for One-Group Slab-Geometry Discrete Ordinates Problems with no Spatial Truncation Error, Nuclear Science \& Engineering, Vol. 104, pp. 199-208.

Chandrasekhar, S., 1950, Radiative Transfer, Oxford University Press, London.

de Abreu, M. P., and Barros, R. C., 1994, An Analytical Reconstruction Scheme for the Dominant Solution of One-Speed Slab-Geometry $\mathrm{S}_{\mathrm{N}}$ Eigenvalue Problems, In: The Fifth Brazilian Nuclear Energy Society Meeting, Vol. I, Rio de Janeiro, Brazil, pp. 201-205.

de Abreu, M. P., 1998, On the Spectrum of the One-Speed Slab-Geometry Discrete Ordinates Operator in Neutron Transport Theory, Annals of 
Nuclear Energy, Vol. 25, pp. 1209-1219.

de Abreu, M. P., 2003, A Mathematical

Method for Solving Mixed Problems in Multislab

Radiative Transfer, Journal of the Brazilian Society of Mechanical Sciences and Engineering (accepted for publication).

de Abreu, M. P., 2004a, A Two-Component Method for Solving Multislab Problems in Radiative Transfer, Journal of Quantitative Spectroscopy and Radiative Transfer, Vol. 85, pp. 311-336.

de Abreu, M. P., 2004b, Mixed SingularRegular Boundary Conditions in Multislab Radiation Transport, Journal of Computational Physics, Vol. 197, pp. 167-185.

Devaux, C., Grandjean, P., Ishiguro, Y., and Siewert, C. E., 1979, On Multi-Region Problems in Radiative Transfer, Astrophysics and Space Science, Vol. 62, pp. 225-233.

Duderstadt, J. J., and Martin, W. R., 1979, Transport Theory, John Wiley \& Sons, New York.

Lewis, E. E., and Miller Jr., W. F., 1993, Computational Methods of Neutron Transport, American Nuclear Society, Lagrange Park, IL, USA.

Siewert, C. E., 2000, A Concise and Accurate Solution to Chandrasekhar's Basic Problem in Radiative Transfer, Journal of Quantitative Spectroscopy and Radiative Transfer, Vol. 64, pp. 109-130.

Thomas, G. E., and Stamnes, K., 1999, Radiative Transfer in the Atmosphere and Ocean, Cambridge University Press, New York. 